\title{
Multi-Dimensional Travelling-Wave Solutions of a Flame Propagation Model
}

\author{
H. Berestycki, B. LARRouturou \& P. L. Lions
}

Communicated by H. BREZIS

\section{Introduction}

In this paper we show the existence of a travelling wave solution of the equation

$$
\frac{\partial u}{\partial t}+\alpha(y) \frac{\partial u}{\partial x_{1}}=\Delta u+g(u),
$$

set in the infinite cylindrical domain $\Sigma=\left\{\left(x_{1}, y\right) \in \mathbb{R} \times \omega\right\}$, where $\omega$ is a bounded and smooth open domain in $\mathbb{R}^{N-1}$. This equation arises in combustion theory: it describes the propagation of a curved premixed flame in the infinite tube $\Sigma$, in the framework of the classical thermo-diffusive model, under the assumption that the Lewis number is equal to unity.

Referring to [3], [4], [13], [15] e.g. for more details, we simply recall here the equations of the thermo-diffusive model, which is derived in the framework of the well known constant-density approximation. We consider a curved flame propagating in the infinite cylindrical tube $\Sigma=\mathbb{R} \times \omega \subset \mathbb{R}^{N}$. For $x \in \Sigma$, we write $x=\left(x_{1}, y\right)$ with $x_{1} \in \mathbb{R}$ and $y \in \omega$. With the assumption of a single one-step chemical reaction $\mathscr{R} \rightarrow \mathscr{P}$, the equations of the thermo-diffusive model are

$$
\begin{aligned}
& u_{t}+\alpha(y) u_{x_{1}}=\Delta u+f(u) v, \\
& v_{t}+\alpha(y) v_{x_{1}}=\frac{\Delta v}{\mathrm{Le}}-f(u) v \quad \text { in } \Sigma ;
\end{aligned}
$$

Here $u$ is the normalized temperature and $v$ is the mass fraction of the reactant. Moreover, $\alpha(y)$ is the $x_{1}$-component of the velocity field $\vec{V}=(\alpha(y), 0)$ which is given, parallel to the tube walls $\partial \Sigma$ and divergence free. Lastly, the terms $\Delta u$, $\frac{\Delta v}{\mathrm{Le}}$ and $f(u) v$ correspond to the thermal diffusion, the molecular diffusion (the non-dimensional positive parameter Le is the Lewis number of the reactant $\mathscr{R}$ ), and the chemical reaction respectively. 
The travelling-wave solutions $u\left(x_{1}+c t, y\right), v\left(x_{1}+c t, y\right)$ of (1.2) satisfy the equations

$$
\begin{aligned}
& (c+\alpha(y)) u_{x_{1}}=\Delta u+f(u) v, \\
& (c+\alpha(y)) v_{x_{1}}=\frac{\Delta v}{\mathrm{Le}}-f(u) v \quad \text { in } \Sigma,
\end{aligned}
$$

and the following boundary conditions (which are classical in combustion theory):

$$
\begin{aligned}
\frac{\partial u}{\partial v}=0, & \frac{\partial v}{\partial v}=0 \quad \text { on } \partial \Sigma, \\
u(-\infty, y)=0, & v(-\infty, y)=1, \\
u(+\infty, y)=1, & v(+\infty, y)=0 \quad \text { for } y \in \omega .
\end{aligned}
$$

In (1.5), $v$ is the outward unit normal on $\partial \Sigma$. When $L e=1,(1.3)-(1.5)$ obviously imply $u+v=1$. The travelling-wave solutions are then given by a single elliptic equation [we set $g(u)=(1-u) f(u)$ ], namely

$$
(c+\alpha(y)) u_{x_{1}}=\Delta u+g(u) \quad \text { in } \Sigma,
$$

associated with the boundary conditions

$$
\begin{gathered}
\frac{\partial u}{\partial \nu}=0 \quad \text { on } \partial \Sigma, \\
u(-\infty, y)=0, \quad u(+\infty, y)=1 \quad \text { for } y \in \omega .
\end{gathered}
$$

This is the problem which we investigate in this paper. In (1.6)-(1.8), both $u \in \mathscr{C}^{2}(\bar{\Sigma})$ and $c \in \mathbb{R}$ are unknown. We will use the following hypotheses [(1.11) corresponds to an ignition temperature assumption]:

$$
\alpha \text { is a continuous function from } \bar{\omega} \text { into } \mathbb{R} \text {, }
$$

$g$ is a Lipschitz-continuous function from $[0,1]$ into $\mathbb{R}_{+}, g(1)=0$,

$$
\exists \theta \in(0,1) \text { such that } g \equiv 0 \text { on }[0, \theta] \text { and } g>0 \text { on }(\theta, 1) \text {. }
$$

With the notation $\langle\alpha\rangle=\frac{1}{|\omega|} \int_{\omega} \alpha(y) d y$, we can now state our main result:

Theorem 1.1. For any functions $\alpha$ and $g$ satisfying (1.9)-(1.11), there exists $a$ solution $(u, c)$ of $(1.6)-(1.8)$. This solution satisfies

$$
\begin{gathered}
0<u<1 \quad \text { in } \bar{\Sigma}, \\
u_{x_{1}}>0 \quad \text { in } \bar{\Sigma}, \\
\langle\alpha\rangle+c>0 . \quad \square
\end{gathered}
$$

Remark 1.2. If $(u, c)$ is a solution of (1.6)-(1.8), it is clear from the classical elliptic estimates that $u \in W_{\mathrm{loc}}^{2, p}(\Sigma)$ for all $p \in(1,+\infty)$ (see AGmon, Douglis 
\& NIRENBERG [1]). Moreover, from classical Schauder estimates, $u \in \mathscr{C}^{2}(\Sigma)$ and therefore satisfies (1.6) in the usual sense if the function $\alpha(y)$ is Hölder-continuous.

Remark 1.3. Uniqueness of the solution of (1.6)-(1.8) has been proved by BERESTYCKI \& NIRENBERG [7]. Assuming that $g$ is a Lipschitz-continuous function in $[0,1]$, is $\mathscr{C}^{2}$ in a neighborhood of 1 and satisfies $g^{\prime}(1)<0$, it is shown in [7] that if $(u, c)$ and $\left(u^{\prime}, c^{\prime}\right)$ are two solutions of (1.6)-(1.8), then $c=c^{\prime}$ and $u\left(x_{1}, y\right)=u^{\prime}\left(x_{1}+\hat{x}_{1}, y\right)$ for all $\left(x_{1}, y\right) \in \bar{\Sigma}$, for some $\hat{x}_{1} \in \mathbb{R}$. Furthermore, that any solution $u$ of (1.6)-(1.8) (with arbitrary $c \in \mathbb{R}$ ) is monotone has also been established by BERESTYCKI \& NIRENBERG [6], [7] under the assumptions (1.9)-(1.11). This last statement of monotonicity has been extended to a somewhat more general class of nonlinearities by C. M. Lr [14].

The existence of a solution $(u, c)$ of (1.6)-(1.8) for any functions $\alpha$ and $g$ satisfying (1.9)-(1.11) is proved in Section 2 below.

Problem (1.6)-(1.8) had been previously investigated by the first two authors in [3], under the additional assumption essentially that $\alpha$ does not differ much from a constant. More precisely, under the assumption that

$$
\left(\max _{y \in \bar{\omega}} \alpha(y)-\min _{y \in \bar{\omega}} \alpha(y)\right)\left(\langle\alpha\rangle-\min _{y \in \bar{\omega}} \alpha(y)\right)<2 \int_{0}^{1} g(s) d s,
$$

it was shown in [3] that there exists a solution $(u, c)$ to $(1.6)-(1.8)$, and that this solution satisfies

$$
c+\min _{y \in \bar{\omega}} \alpha(y)>0
$$

This inequality, which is derived from the additional assumption (1.15), is crucially used in [3] to derive some a priori estimates. In contrast with this situation, for the solutions we construct here, $c+\alpha(y)$ may in general change sign in the domain $\omega$. This phenomenon may be interpreted as an "inversion of the velocity field". Indeed, (1.2)-(1.3) show that $c+\alpha(y)$ is the mixture velocity in the reference frame $R_{f}$ in which the solution is stationary $\left(R_{f}\right.$ is a reference frame attached to the flame and moves with the velocity $-c$ with respect to the original reference frame $R_{0}$ ). Then, (1.16) means that, at every point, the velocity in the reference frame $R_{f}$ points from left to right, i.e. from the fresh mixture towards the burnt gases, a physically natural situation. But, for solutions satisfying

$$
c+\min _{y \in \overline{\bar{\omega}}} \alpha(y)<0,
$$

there are regions of the tube (where $c+\alpha(y)<0$ ) where the velocity is directed in the opposite way, from the burnt gases towards the fresh mixture! It is important to realize here that this non-classical situation is by no means unphysical: it has indeed been known for a long time (not for a flame in a tube, but in other geometrical configurations, such as for a counterflow diffusion flame; see WiLLiams $[15$, p. 418]) that the mixture velocity in the neighborhood of the flame may be pointing from the burnt gases towards the fresh mixture. This simply means that 
the convective effects are locally dominated by the diffusive effects. Moreover, in these conditions, (1.14) says that the average velocity in the reference frame $R_{f}$ is necessarily positive.

Several results in this direction are shown in Section 3. We prove there that, the function $g$ corresponding to the reaction term being given, one can choose the function $\alpha$ (far enough from a constant) so that the corresponding solution $(u, c)$ of $(1.6)-(1.8)$ actually satisfies $(1.17)$ (such travelling wave solutions have been numerically computed in [2]). Moreover, we show that condition (1.15) is, in some sense, optimal to insure that the inversion of the velocity field does not occur (i.e. that property (1.16) is fulfilled).

The existence of travelling fronts in two dimensions but with different boundary conditions has been examined in only one other work to our knowledge. In an interesting paper, R. GARDNER [8] establishes the existence of $c$ and $u$ solution of problems of the type

$$
\begin{gathered}
-\Delta u+c u_{x}=f(u) \quad \text { in } \tilde{\Sigma}, \\
u(x, 0)=u(x, L)=0, \\
u(-\infty, y)=0, \quad u(+\infty, y)=\phi(y) .
\end{gathered}
$$

Here, $\tilde{\Sigma}=\left\{(x, y) \in \mathbb{R}^{2}, 0<y<L\right\}, f(u)=u(1-u)(u-\beta)$ with $0<\beta<\frac{1}{2}$, and $\phi$ is the maximal positive solution of $\phi^{\prime \prime}+f(\phi)=0, \phi(0)=\phi(L)=0$ (for $L$ sufficiently large). R. GARDNER [8] uses a method relying on the Conley index.

\section{Proof of Existence}

In this section we prove Theorem 1.1. As in [3], this proof reduces to studying an analogous problem posed on the bounded cylindrical domain $R_{a}=(-a, a)$ $\times \omega$ for $a \in \mathbb{R}$ and then in examining the passage to the limit as $a \rightarrow+\infty$. Solving the problem in $R_{a}$ is essentially the same here as in [3]. However [because the solution here does not necessarily satisfy (1.16)], the arguments in [3] would fail to yield the limit as $a \rightarrow+\infty$. Hence, we need here another approach to the derivation of the a priori estimates and to the limiting procedure.

We first consider the problem

$$
(c+\alpha(y)) u_{x_{1}}=\Delta u+g(u) \quad \text { in } R_{a},
$$

with the mixed boundary conditions:

$$
\begin{gathered}
\frac{\partial u}{\partial \nu}=0 \quad \text { on }(-a, a) \times \partial \omega, \\
u(-a, y)=0, \quad u(+a, y)=1 \quad \text { for } y \in \omega .
\end{gathered}
$$

To this system we add the following normalization condition which we trade against the freedom to choose $c$ :

$$
\max _{\left(x_{1}, y\right) \in \bar{R}_{a}, x_{1} \leq 0} u\left(x_{1}, y\right)=\theta .
$$


The role of this condition (and also analogous normalization conditions) is discussed in Berestycki \& LARrouturou [3] and in BerestyCKI, NicolaenKo \& SCHEURER [5].

On problem (2.1)-(2.4), we are going to prove

Proposition 2.1. Under the assumptions (1.9)-(1.11) and for any $a>0$, there is a solution $(u, c)=\left(u_{a}, c_{a}\right)$ of problem $(2.1)-(2.4)$.

The proof follows the steps of the one given in [3] with a few minor modifications. For the sake of completeness, we repeat it here. It rests on the following $a$ priori estimates (in what follows we always assume that the definition of $g$ is extended to all of $\mathbb{R}$ by setting $g(s) \equiv 0$ for $s \notin[0,1])$ :

Lemma 2.2. Suppose $g \leqq M$ on $[0,1]$ and $\alpha_{0} \leqq \alpha(y) \leqq \alpha_{1}$ for all $y \in \bar{w}$. Then there is a constant $K$, depending only on $a, \alpha_{0}, \alpha_{1}$ and $M$, such that any solution $(u, c)$ of (2.1)-(2.4) satisfies

and:

$$
|c| \leqq K
$$

$$
\|u\|_{\mathscr{C}^{1}\left(\bar{R}_{a}\right)} \leqq K .
$$

Proof of Lemma 2.2. Since $g(s)=0$ outside the interval $[0,1]$, it follows from the maximum principle that $0<u<1$ in $R_{a}$. Hence, by a result of BERESTYCKI \& NiRENBERG [6, Theorem 4.1], we know that $u_{x_{1}}>0$ in $(-a, a) \times \bar{\omega}$ (actually, it is assumed in [6, Theorem 4.1] that $u$ satisfies Dirichlet data on the whole boundary of $R_{a}$; but of course the same result holds under the present conditions). Using this information, we infer some inequalities from the maximum principle. Let $\alpha_{0}$ and $\alpha_{1}$ be such that $\alpha_{0} \leqq \alpha(y) \leqq \alpha_{1}$ for all $y \in \bar{\omega}$, and denote $z_{0}, z_{1}$ the solutions of the following ordinary differential equations:

$$
\begin{array}{ll}
-z_{0}^{\prime \prime}+\left(c+\alpha_{1}\right) z_{0}^{\prime}=0 & \text { in }(-a, a), \\
-z_{1}^{\prime \prime}+\left(c+\alpha_{0}\right) z_{1}^{\prime}=M & \text { in }(-a, a),
\end{array}
$$

together with the boundary conditions

$$
z_{i}(-a)=0, \quad z_{i}(+a)=1 \quad \text { for } i=0,1 .
$$

The maximum principle then yields

$$
z_{0}\left(x_{1}\right) \leqq u\left(x_{1}, y\right) \leqq z_{1}\left(x_{1}\right) \quad \text { in } R_{a} ;
$$

for instance, the first inequality in (2.10) follows from the relation

$$
-\Delta\left(u-z_{0}\right)+\left(c+\alpha_{1}\right)\left(u-z_{0}\right)_{x_{1}}=g(u)+\left(\alpha_{1}-\alpha(y)\right) u_{x_{1}} \geqq 0 .
$$

Now, since, for fixed $a>0$

$$
\lim _{c \rightarrow-\infty} z_{0}(0)=1 \quad \text { and } \quad \lim _{c \rightarrow+\infty} z_{1}(0)=0
$$


the condition $z_{0}(0) \leqq \theta \leqq z_{1}(0)$ which follows from (2.4) gives the a priori

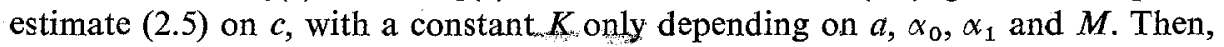
since $0<u<1$ and $c$ is bounded, we immediately derive by the elliptic estimates a $\mathscr{C}^{1}$ bound on $u:\|u\|_{\mathscr{C}^{1}}\left(\bar{R}_{a}\right) \leqq K$.

Proof of Proposition 2.1. Consider the space $E=\mathscr{C}^{1}\left(\bar{R}_{a}\right)$ and $\mathscr{E}=E \times \mathbb{R}$. For $(v, c) \in \mathscr{E}$ and for $\tau \in[0,1]$, let $u=\phi_{\tau}(v, c)$ be the unique solution of

$$
-\Delta u+(c+a(y)) u_{x_{1}}=\tau g(v) \text { in } R_{a},
$$

with $u$ satisfying (2.2) and (2.3). Hence problem (2.1)-(2.3) has been translated into the equation $u=\phi_{1}(u, c)$. Next, let

$$
h_{\tau}(v, c)=\max _{\left(x_{1}, y\right) \in \bar{R}_{a}, x_{1} \leqq 0} \phi_{\tau}(v, c) .
$$

Thus our problem (2.1)-(2.4) with unknowns $u$ and $c$ is now written as a fixed point equation in the space $\mathscr{E}$ :

$$
\begin{aligned}
& u=\phi_{3}(u, c), \\
& h_{1}(u, c)=\theta .
\end{aligned}
$$

This problem is of the form $(u, c)-\mathscr{F}_{1}(u, c)=0$ where, for $\tau \in[0,1]$

$$
\mathscr{F}_{r}(u, c)=\left(\phi_{\tau}(u, c), c-h_{\tau}(u, c)+\theta\right) .
$$

Clearly the mapping $(\tau,(u, c)) \rightarrow \mathscr{F}_{\tau}(u, c)$ from $[0,1] \times \mathscr{E}$ into $\mathscr{E}$ is continuous and compact. Now, let the reals $\alpha_{0}$ and $\alpha_{1}$ be given by

$$
\alpha_{0}=\min \left(\min _{y \in \bar{\omega}} \alpha(y), 0\right), \quad \alpha_{1}=\max \left(\max _{y \in \bar{\omega}} \alpha(y), 0\right),
$$

so that

$$
\alpha_{0} \leqq \tau \alpha(y) \leqq \alpha_{1}
$$

for all $y \in \bar{\omega}$ and $\tau \in[0,1]$. Let $K$ be the constant introduced in Lemma 2.2; $K$ depends on $a, \alpha_{0}, \alpha_{1}$ and $M$. Since $\tau g(s) \leqq M$, we know by the estimates in Lemma 2.2 that $(u, c)-\mathscr{F}_{x}(u, c) \neq 0$ for all $(u, c) \in \partial \Omega$ and all $\tau \in[0,1]$, where

$$
\Omega=\left\{(u, c) \in \mathscr{E},\|u\|_{\mathscr{G}^{1}\left(\vec{R}_{a}\right)} \leqq K,|c| \leqq K\right\} .
$$

Hence the Leray-Schauder degree $d\left(I-\mathscr{F}_{1}, \Omega, 0\right)$ is well defined and

$$
d\left(I-\mathscr{F}_{1}, \Omega, 0\right)=d\left(I-\mathscr{F}_{0}, \Omega, 0\right)
$$

by homotopy invariance. Now for $\tau=0, \phi_{0}(u, c)$ is independent of $u$. We perform a new homotopy by substituting $\tau \alpha(y)$ for $\alpha(y)$ in the definition (2.13) of $\phi_{\tau}$ and subsequently in the definition (2.14) of $h_{\tau}$. Continuity and compactness are left unchanged as are our estimates in Lemma 2.2 by the choice we have made of $\alpha_{0}$ and $\alpha_{1}$. Therefore, by homotopy invariance,

$$
d\left(I-\mathscr{F}_{1}, \Omega, 0\right)=d\left(I-\mathscr{F}_{1}^{*}, \Omega, 0\right),
$$


where $\mathscr{F}_{1}^{*}(u, c)=\left(\psi_{c}, c-\hat{h}(c)+\theta\right)$, with $\psi_{c}=\psi_{c}\left(x_{1}\right)$ the solution of

$$
\begin{gathered}
-\psi_{c}^{\prime \prime}+c \psi_{c}^{\prime}=0 \quad \text { in }(-a, a), \\
\psi_{c}(-a)=0, \quad \psi_{c}(+a)=1,
\end{gathered}
$$

and:

$$
\hat{h}(c)=\psi_{c}(0)
$$

But $\hat{h}(c)=\frac{1-e^{-c a}}{e^{c a}-e^{-c a}}$, and the equation $(u, c)=\mathscr{F}_{1}^{*}(u, c)$ uniquely determines $c$ and consequently $\left(u\left(c=c^{*}\right)\right) \in(-K, K)$ and $\left.u=\psi_{c^{*}}\right)$. Then, by homotopy invariance, the degree (2.21) is the same as the degree of the mapping:

$$
(u, c) \rightarrow\left(u-\psi_{c^{*}}, \hat{h}(c)-\theta\right) .
$$

By using the product property of the degree we obtain, letting $B_{K}$ stand for the ball of radius $K$ in $E$, the equations

$$
d\left(I-\mathscr{F}_{1}, \Omega, 0\right)=d\left(I-\psi_{c^{*}}, B_{K}, 0\right), d(\hat{h}(c)-\theta,(-K, K), 0) .
$$

Since $\psi_{c^{*}} \in B_{K}$ and $\hat{h}$ is decreasing with $\hat{h}(-K)>0, \hat{h}(K)<0$, we finally conclude that

$$
d\left(I-\mathscr{F}_{1}, \Omega, 0\right)=-1 .
$$

Hence there is a solution $(u, c)$ of problem (2.1)-(2.4), which completes the proof of Proposition 2.1.

Remark 2.3. Under the same assumptions as in Remark 1.3, uniqueness of the solution $(u, c)$ of $(2.1)-(2.4)$ has been proved by BERESTYCKI \& NIRENBERG [7].

Now comes the newer part in the proof of Theorem 1.1. We will now derive the limit of the solution $\left(u_{a}, c_{a}\right)$ of (2.1)-(2.4) when $a \rightarrow+\infty$.

\section{Proof of Theorem 1.1, divided into several steps}

Step 1: A priori estimate on $c_{a}$.

Lemma 2.4. There is a constant $K>0$, independent of $a$, such that for any $a \geqq 1$ the solution $\left(u_{a}, c_{a}\right)$ of (2.1)-(2.4) satisfies

$$
\left|c_{a}\right| \leqq K
$$

Proof. We recall from the proof of Lemma 2.2 that the function

$$
z_{0}\left(x_{1}\right)=\frac{e^{\left(c_{a}+\alpha_{1}\right) x_{1}}-e^{-\left(c_{a}+\alpha_{1}\right) a}}{2 \sinh \left(\left(c_{a}+\alpha_{1}\right) a\right)}
$$


satisfies $z_{0}\left(x_{1}\right) \leqq u_{a}\left(x_{1}, y\right)$ on $\bar{R}_{a}$. Hence $z_{0}(0) \leqq \theta$, which implies $\left(c_{a}+\alpha_{1}\right) a$ $\geqq-K^{\prime}$ for some constant $K^{\prime}>0$, or

$$
c_{a} \geqq-\alpha_{1}-\frac{K^{\prime}}{a} \text {. }
$$

Let us now derive an upper bound for $c_{a}$. To this end, we use the solution $z\left(x_{1}\right)$ of

$$
\begin{gathered}
-z^{\prime \prime}+\left(c_{a}+\alpha_{0}\right) z^{\prime}=M H\left(x_{1}\right) \quad \text { in }(-a, a), \\
z(-a)=0, \quad z(+a)=1,
\end{gathered}
$$

with $M$ defined as before and $H\left(x_{1}\right)=1$ if $x_{1}>0$ and 0 otherwise. Clearly $z\left(x_{1}\right) \geqq u_{a}\left(x_{1}, y\right)$ on $\bar{R}_{a}$ and therefore $z(0) \geqq \theta$. A direct computation then yields the estimate $c_{a} \leqq K$ for all $a \geqq 1$.

Step 2: Existence of a limit.

From the estimate (2.27) on $c_{a}$ we see by the classical elliptic estimates (see Agmon, Douglis \& NiRENBERG [1]) that, for any $p>1, u_{a}$ is bounded in the $W^{2, p}$ norm in any rectangle $\left(x_{1}, x_{1}+1\right) \times \omega$ contained in $\bar{R}_{a}$, and this holds independently of $a$ and $x_{1}$. In particular, there is a $K>0$ independent of $a$ such that

$$
\left\|u_{a}\right\|_{\mathscr{G}^{1}\left(\bar{R}_{a}\right)} \leqq K
$$

for all $a \geqq 1$.

Moreover, we can find a sequence $a_{n} \rightarrow+\infty$ such that $c_{a_{n}} \rightarrow c$ in $\mathbb{R}$ and $u_{a_{n}} \rightarrow u$ locally in $\mathscr{C}^{1}$ norm. Obviously we obtain a solution of the equation in $\Sigma$

$$
(c+\alpha(y)) u_{x_{1}}=\Delta u+g(u),
$$

which satisfies

$$
\frac{\partial u}{\partial v}=0 \quad \text { on } \partial \Sigma
$$

Furthermore, since $\frac{\partial u_{a}}{\partial x_{1}}>0$ and $0<u_{a}<1$ for all $a>0$, we obtain $u_{x_{1}} \geqq 0$ and $0 \leqq u \leqq 1$ in $\bar{\Sigma}$.

From now on in this section, $(u, c)$ will always denote the limit of $\left(u_{a_{n}}, c_{a_{n}}\right)$. It now remains to study the limits of $u\left(x_{1}, y\right)$ as $x_{1} \rightarrow \pm \infty$. In particular, we wish to prevent $u_{a}$ from converging locally to some constant $\varrho \in[0,0] \cup\{1\}$.

Step 3: Energy estimates

Lemma 2.5. The following integrals are bounded

$$
\int_{\Sigma} g(u)<+\infty, \quad \int_{\Sigma}|\nabla u|^{2}<+\infty \text {. }
$$


Here and hereafter the measure $d x_{1} d y$ is understood to refer to integrals taken over $\Sigma$ or parts of $\Sigma$.

Proof. (the same as in [3] and repeated here): On $\mathbb{R}_{-} \times \omega$, we have $g(u) \equiv 0$ since $u \leqq \theta$. Besides, for $z>0$, let $\left.Q_{z}=\right] 0, z\left[\times \omega\right.$ and $U(z)=\int_{\omega} u(z, y) d y$.
Integrating equation (1.6) on $Q_{z}$, we have

$$
\int_{Q_{z}} g(u)=A(z)-A(0),
$$

with

$$
A(z)=c \int_{\omega} \alpha(y) u(z, y) d y-U^{\prime}(z) .
$$

If $\int_{Q_{z}} g(u) \rightarrow+\infty$ when $z \rightarrow+\infty$, then $U^{\prime}(+\infty)=-\infty$, which is impossible since $U$ is bounded. For the second integral in (2.32), we multiply (1.6) by $u$ before integrating on $Q_{z}$, and we conclude in the same manner.

Step 4: Existence of limits as $x_{1} \rightarrow \pm \infty$

Since $u_{x_{1}} \geqq 0$, we know that the limits $\lim _{x_{1} \rightarrow \pm \infty} u\left(x_{1}, y\right)=\beta_{ \pm}(y)$ exist. By considering the sequence of functions

$$
u_{j}^{ \pm}\left(x_{1}, y\right)=u\left(x_{1} \pm j, y\right)
$$

for $\left(x_{1}, y\right)$ in the fixed domain $R_{1}$, we derive from the classical elliptic estimates that $u_{j}^{ \pm} \rightarrow \beta_{ \pm}$in the $\mathscr{C}^{1}$ sense. Since $\int_{\Sigma}|\nabla u|^{2}<+\infty$, we find that, necessarily, $\nabla \beta_{ \pm}=0$, i.e. $\beta_{+}$and $\beta_{\ldots}$ are constants. Moreover, since $u$ is monotone in the $x_{1}$ direction,

$$
0 \leqq \beta_{-} \leqq \theta=\max _{y \in \bar{\omega}} u(0, y) \leqq \beta_{+} \leqq 1 .
$$

Again using the uniform convergence of $u_{j}^{+}$to $\beta_{+}$and the finiteness of $\int_{\Sigma} g(u)$, we infer that $g\left(\beta_{+}\right)=0$; thus, either $\beta_{+}=\theta$, or $\beta_{+}=1$.

Step 5: The case $\beta_{4}=\theta$.

Lemma 2.6. If $\beta_{+}=\theta$, then $\beta_{-}=\theta$ and $u \equiv \theta$.

Proof. If $\beta_{+}=\theta$, then $u \leqq \theta$ and $g(u) \equiv 0$. Integrating the equation (1.6) over the domain $R_{m}$ and letting $m \rightarrow+\infty$, we find (because $\lim _{x_{1} \rightarrow \pm \infty}\left|\nabla u\left(x_{1}, y\right)\right|$ $=0$ uniformly for $y \in \omega$ from Step 4)

$$
\left(\beta_{+}-\beta_{-}\right)(c+\langle\alpha\rangle)=0,
$$


where $\langle\alpha\rangle=\frac{1}{|\omega|} \int_{\omega} \alpha(y) d y$. Similarly, multiplying the equation by $u$, we find
that

$$
-\int_{\Sigma}|\nabla u|^{2}-\frac{|\omega|}{2}\left(\beta_{+}^{2}-\beta_{-}^{2}\right)(c+\langle\alpha\rangle)=0 .
$$

Hence, by use of (2.37), it follows that

$$
\int_{\Sigma}|\nabla u|^{2}=0
$$

and we have proved the claim: $u$ must be constant, and $\beta_{-}=\theta$.

Step 6: An estimate for $c+\langle\alpha\rangle$.

We will now show that $c+\langle\alpha\rangle$ is bounded away from 0 by a positive number. We first need the next conclusion about the solution $\left(u_{a}, c_{a}\right)$ of $(2.1)-(2.4)$ :

Lemma 2.7. There is a constant $\delta>0$, independent of a such that for any $a \geqq 1$ the solution $\left(u_{a}, c_{a}\right)$ of $(2.1)-(2.4)$ satisfies:

$$
\int_{R_{a}} g\left(u_{a}\right) \geqq \delta>0
$$

Proof. Choose a number $\lambda$ with $\theta<\lambda<1$. Let $a \geqq 1$. For some $x_{0} \in(0, a)$ and $y_{0} \in \omega$ we have $u_{a}\left(x_{0}, y_{0}\right)=\lambda$. Since $\left|\nabla u_{a}\right| \leqq K$ in $\bar{R}_{a}$ with $K$ independent of $a$, we see that $a-x_{0} \geqq \frac{1-\lambda}{K}, x_{0} \geqq \frac{\lambda-\theta}{K}$. Then we can find $\eta>0$, $\sigma>0$ and $\varepsilon>0$ independent of $a$ such that

$$
g\left(u\left(x_{1}, y\right)\right) \geqq \sigma \quad \text { for all }\left(x_{1}, y\right) \in \Sigma \cap B_{\eta}\left(x_{0}, y_{0}\right),
$$

and

$$
\left|\Sigma \cap B_{\eta}\left(x_{0}, y_{0}\right)\right| \geqq \varepsilon
$$

We thus obtain

$$
\int_{R_{a}} g\left(u_{a}\right) \geqq \varepsilon \sigma
$$

which completes the proof.

A consequence of Lemma 2.7 is the following result.

Lemma 2.8. Let $\delta>0$ be the constant defined in Lemma 2.7. Then $c$ satisfies

$$
\langle\alpha\rangle+c\rangle \frac{\delta}{|\omega|}
$$


Proof. We will separately consider both cases $\beta_{+}=\theta$ and $\beta_{+}=1$.

a) Assume first that $\beta_{+}=\theta$, and integrate the equation (2.1) satisfied by $u_{a_{n}}$ on the domain $R_{a_{n}}^{+}=\left(0, a_{n}\right) \times \omega$. We get

$$
\begin{aligned}
\int_{R_{a_{n}}^{+}} g\left(u_{a_{n}}\right)= & \left(c_{a_{n}}+\langle\alpha\rangle\right)|\omega|-\int_{\omega}\left(c_{a_{n}}+\alpha(y)\right) u_{a_{n}}(0, y) d y \\
& \left.-\int_{\omega}^{\partial u_{a_{n}}} \partial a_{n}, y\right) d y+\int_{\omega} \frac{\partial u_{a_{n}}}{\partial x_{1}}(0, y) d y .
\end{aligned}
$$

But we know from Lemma 2.6 that $u_{a_{n}}$ converges in the $\mathscr{C}^{1}$ sense to $\theta$ on any compact subset of $\bar{\Sigma}$. Then the third and fifth terms in (2.45) converge to $(c+\langle\alpha\rangle) \theta|\omega|$ and 0 respectively. Moreover, using the fact that $\frac{\partial u_{a_{n}}}{\partial x_{1}}>0$ and Lemma 2.7, we
obtain

$$
(1-\theta)|\omega|(c+\langle\alpha\rangle) \geqq \delta,
$$

whence (2.44).

b) Assuming now that $\beta_{+}=1$, we can argue as in the proof of Lemma 2.7 to show that $u$ (and not $u_{a}$ ) also satisfies

$$
\int_{\Sigma} g(u) \geqq \delta>0 .
$$

Integrating now equation (1.6) on all of $\Sigma$ (as in the proof of Lemma 2.6), we find that

$$
|\omega|\left(\beta_{+}-\beta_{-}\right)(c+\langle\alpha\rangle)=\int_{\Sigma} g(u),
$$

from which (2.44) again follows.

Step 7: Conclusion.

We can now conclude the proof of Theorem 1.1, using the following lemma.

Lemma 2.9. Under the assumption $\langle\alpha\rangle+\gamma>0$, there are a unique $\lambda>0$ and a corresponding "eigenfunction" $\Psi=\Psi(y)$ (which is strictly positive in $\bar{\omega}$ ) that solve the following problem:

$$
\begin{gathered}
-\Delta_{y} \Psi+\lambda(\gamma+\alpha(y)) \Psi=\lambda^{2} \Psi \text { in } \omega \\
\frac{\partial \Psi}{\partial v}=0 \quad \text { on } \partial \omega . \square
\end{gathered}
$$

This result is a particular case of Theorem 3.4 in BERESTYCKI \& NIRENBERG [7]. We refer the reader to [7] for the complete proof. 
Here we use Lemma 2.9 to define a barrier function for $u$. First we choose a real $\gamma$ in such a way that $\langle\alpha\rangle+\gamma>0$ and $\gamma\left\langle c_{a}\right.$ for large $a$ (i.e. $\gamma=c-\varepsilon$ for some small $\varepsilon>0$ ). Since $\Psi$ is defined up to a multiplicative constant, we may as well assume that $\Psi(y) \geqq \theta$ on $\bar{\omega}$. Then we consider the function $\Phi$ defined by

$$
\Phi\left(x_{1}, y\right)=e^{\lambda x_{1}} \Psi(y) .
$$

Since $\left(u_{a}\right)_{x_{1}} \geqq 0$, for large $a$

$$
-\Delta\left(\Phi-u_{a}\right)+(\gamma+\alpha(y))\left(\Phi-u_{a}\right)_{x_{1}}=\left(c_{a}-\gamma\right)\left(u_{a}\right)_{x_{1}} \geqq 0 \quad \text { in }(-a, 0) \times \omega,
$$

with the boundary inequalities

$$
\left(\Phi-u_{a}\right)(-a, y) \geqq 0, \quad\left(\Phi-u_{a}\right)(0, y) \geqq 0 \quad \text { for } y \in \omega,
$$

and

$$
\frac{\partial\left(\Phi-u_{a}\right)}{\partial v}=0 \quad \text { on }(-a, 0) \times \partial \omega
$$

The maximum principle then yields

$$
u_{a}\left(x_{1}, y\right) \leqq \Phi\left(x_{1}, y\right)
$$

for all $-a \leqq x_{1} \leqq 0$ and $y \in \omega$. Hence

$$
u\left(x_{1}, y\right) \leqq e^{\lambda x_{1}} \Psi(y)
$$

for all $x_{1} \leqq 0$ and $y \in \omega$. Since $\lambda>0$, this shows that $\beta_{-}=0$; Step 5 then shows that $\beta_{+}$cannot be equal to $\theta$. Thus $\beta_{+}=1$ and the proof of Theorem 1.1 is complete.

\section{Inversion of the Velocity Field}

As we said in the introduction, we consider in this section the inversion of the velocity field $c+\alpha(y)$ in the reference frame $R_{f}$ attached to the flame; that is, we examine the sign of $c+\min _{y \in \bar{\omega}} \alpha(y),(u, c)$ being the solution of (1.6)-(1.8).

The existence of solutions with $c+\min _{y \in \bar{\omega}} \alpha(y)<0$ rests on the following observation concerning a sequence $\left(u_{n}, c_{n}\right)$ of solutions of the problem

$$
\begin{gathered}
\left(c_{n}+\alpha_{n}(y)\right) \frac{\partial u_{n}}{\partial x_{1}}=\Delta u_{n}+g\left(u_{n}\right) \quad \text { in } \Sigma, \\
\frac{\partial u_{n}}{\partial v}=0 \quad \text { on } \partial \Sigma, \\
u_{n}(-\infty, y)=0, \quad u_{n}(+\infty, y)=1 \quad \text { for } y \in \omega, \\
\max _{\left(x_{1}, y\right) \in \bar{\Sigma}, x_{1} \leqq 0} u_{n}\left(x_{1}, y\right)=\theta .
\end{gathered}
$$


Proposition 3.1. Let $\alpha_{0}$ and $\alpha_{1}$ be two real numbers with $\alpha_{0}<\alpha_{1}$. For $n \geqq 2$, let $\alpha_{n}$ be a continuous function on $\bar{\omega}$ such that $\lim _{n \rightarrow+\infty} \alpha_{n}(y)$ exists for almost all $y$ in $\omega$ :

$$
\lim _{n \rightarrow+\infty} \alpha_{n}(y)=\alpha(y) \quad \text { a.e. in } \omega,
$$

and that, for all $n \geqq 2$,

$$
\alpha_{0} \leqq \alpha_{n}(y) \leqq \alpha_{1} \quad \text { in } \bar{\omega}
$$

Let $g$ satisfy the hypotheses (1.10)-(1.11), and let $\left(u_{n}, c_{n}\right)$ be the solution of (3.1)(3.4). Then $\left(u_{n}, c_{n}\right)$ converges in $\mathscr{C}_{\mathrm{loc}}^{1}(\bar{\Sigma}) \times \mathbb{R}$ towards $(u, c)$ satisfying $(1.6)-(1.8)$.

Proof. From Section 2, the estimates on $u_{n}$ in $W^{2, p}\left(R_{b}\right)$ for any positive $b$, on $c_{n}$ in $\mathbb{P}$ and the estimate $\left.\left\langle\alpha_{n}\right\rangle+c_{n}\right\rangle \frac{\delta}{|\omega|}$ only depend on $\alpha_{0}, \alpha_{1}$ and $g$ (and not on $n)$. We can therefore extract a subsequence $\left(u_{n_{k}}, c_{n_{k}}\right)$ which converges in $\mathscr{C}_{\text {loc }}^{1}(\bar{\Sigma})$ $\times \mathbb{R}$ to $(u, c)$. Then $(u, c)$ satisfies the boundary condition $(1.7)$ on $\partial \Sigma$ and

$$
\max _{\left(x_{1}, y\right) \in \bar{\Sigma}, x_{1} \leqq 0} u\left(x_{1}, y\right)=\theta, \quad u_{x_{1}} \geqq 0
$$

Moreover (using Lebesgue's bounded convergence theorem), we easily see that $(u, c)$ satisfies $(1.6)$ in the sense of the distributions in $\Sigma$. Lastly, we have $u(+\infty, y)$ $=\beta_{+} \in\{\theta, 1\}$ and $u(-\infty, y)=\beta_{-} \in[0, \theta]$ for all $y \in \omega$ as in Steps 3 and 4 above. But we also know that

$$
\langle\alpha\rangle+c=\lim _{n \rightarrow+\infty}\left(\left\langle\alpha_{n_{k}}\right\rangle+c_{n_{k}}\right) \geqq \frac{\delta}{|\omega|}>0 .
$$

Arguing as in Step 7 above, this shows that $\beta_{-}=0, \beta_{+}=1$ and it completes the proof; by use of the uniqueness theorem of Remark 1.3 above, it is classical to show that the whole sequence $\left(u_{n}, c_{n}\right)$ converges to $(u, c)$.

It easily follows from Proposition 3.1 that there are solutions $(u, c)$ of $(1.6)-$ (1.8) having an inversion of the velocity field, i.e. such that $c+\alpha(y)$ changes sign in $\omega$, or, equivalently,

$$
c+\min _{y \in \bar{\omega}} \alpha(y)<0
$$

Indeed, starting with a solution $(u, c)$ for some $\alpha$, one can modify $\alpha$ about some point $y_{0} \in \omega$ without changing $c$ much. Hence one obtains a solution with $c+\alpha\left(y_{0}\right)<0$. This procedure is detailed below.

Proposition 3.2. Let $g$ satisfy (1.10)-(1.11). Then there is a continuous function $\alpha(y)$ such that the corresponding solution $(u, c)$ of $(1.6)-(1.8)$ satisfies $(1.17)$. 
Proof. Let $\alpha_{1}$ be a positive real, to be chosen later. Let $y_{0} \in \omega$. For any integer $n$ we define the function $\alpha_{n}$ by

$$
\alpha_{n}(y)= \begin{cases}\alpha_{1} & \text { if }\left|y-y_{0}\right|>\frac{1}{n}, \\ n \alpha_{1}\left|y-y_{0}\right| & \text { if }\left|y-y_{0}\right| \leqq \frac{1}{n} .\end{cases}
$$
For all $n, \alpha_{n}$ is continuous on $\bar{\omega}$; furthermore, $\min _{y \in \bar{\omega}} \alpha_{n}(y)=0, \max _{y \in \bar{\omega}} \alpha_{n}(y)=\alpha_{1}$,
and

$$
\forall y \in \bar{\omega}-\left\{y_{0}\right\}, \quad \lim _{n \rightarrow+\infty} \alpha_{n}(y)=\alpha_{1} .
$$

Then we know from Proposition 3.1 that the solution $\left(u_{n}, c_{n}\right)$ of (3.1)-(3.4) converges as $n \rightarrow+\infty$ to $(u, c)$ satisfying $(1.7)-(1.8)$ and

$$
\left(c+\alpha_{1}\right) u_{x_{1}}=\Delta u+g(u) \text {. }
$$

Besides, it is known (see BerestyCKI, NicolaEnko \& Scheurer [5], Johnson [9], JoHnson \& NACHBAR [10], and KANEL' [11], [12]) that the one-dimensional problem,

$$
\begin{gathered}
-\hat{u}_{x_{1} x_{1}}+\hat{c} \hat{u}_{x_{1}}=g(\hat{u}) \quad \text { in } \mathbb{R}, \\
\hat{u}(-\infty)=0, \quad \hat{u}(0)=\theta, \quad \hat{u}(+\infty)=1
\end{gathered}
$$

(where $\hat{u}=\hat{u}\left(x_{1}\right)$ and $\hat{c}$ are unknown), has a unique solution $(\hat{u}, \hat{c})$. The uniqueness statement recalled in Remark 1.3 then implies that

$$
u\left(x_{1}, y\right)=\hat{u}\left(x_{1}\right) \quad \text { for all }\left(x_{1}, y\right) \in \Sigma,
$$

and

$$
c+\alpha_{1}=\hat{c} .
$$

Observe that $\hat{c}$ only depends on the function $g$. If we choose from the start to take $\alpha_{1}>\hat{c}$, then (3.14) says that $c<0$. For $n$ large enough, we have $c_{n}<0$, whence

$$
c+\min _{y \in \bar{\omega}} \alpha_{n}(y)=c_{n}<0,
$$

which completes the proof. The choice $\alpha(y)=\alpha_{n}(y)$ for $n$ large enough gives a solution of (1.6)-(1.8) which satisfies (1.17), i.e. which exhibits the inversion of the velocity field.

We conclude this section by deriving conditions for the inversion of the velocity field to occur. First we recall from [3] that this inversion never occurs when the condition

$$
\left(\max _{y \in \bar{\omega}} \alpha(y)-\min _{y \in \bar{\omega}} \alpha(y)\right)\left(\langle\alpha\rangle-\min _{y \in \bar{\omega}} \alpha(y)\right)<2 \int_{0}^{1} g(s) d s
$$

is satisfied. 
Proposition 3.3 [3]. Let $\alpha$ and $g$ satisfy (1.10)-(1.11) and (1.15). Then the solution $(u, c)$ of $(1.6)-(1.8)$ satisfies

$$
c+\alpha(y)>0 \quad \forall y \in \bar{\omega} .
$$

Proof. We give here a more direct proof of this property than in [3]. Multiplying (1.6) by $1-u>0$ and integrating in $\Sigma$ (recall from Section 2 that $\lim _{x_{1} \rightarrow \pm \infty}$ $\left|\nabla u\left(x_{1}, y\right)\right|=0$ uniformly for $\left.y \in \omega\right)$, we get

$$
\frac{|\omega|}{2}(c+\langle\alpha\rangle)-\int_{\Sigma}|\nabla u|^{2} \geqq 0 .
$$

Notice that this inequality again shows that $\langle\alpha\rangle+c>0$. Besides, multiplying (1.6) by $u_{x_{1}}$ and integrating in $\Sigma$ yields

$$
|\omega| \int_{0}^{1} g(s) d s=\int_{\Sigma}(c+\alpha(y)) u_{x_{1}}^{2} .
$$
This implies that $|\omega| \int_{0}^{1} g(s) d s \leqq\left(c+\max _{y \in \hat{\omega}} \alpha(y)\right) \int_{\Sigma}|\nabla \boldsymbol{u}|^{2}$, whence, using (3.17),
we have

$$
2 \int_{0}^{1} g(s) d s \leqq\left(c+\max _{y \in \bar{\omega}} \alpha(y)\right) \cdot(c+\langle\alpha\rangle) .
$$
When $(1.15)$ holds, this inequality shows that $c>-\min _{y \in \bar{\omega}} \alpha(y)$, which concludes
the proof. $\square$

Our last result says that condition (1.15) is optimal in the following sense.

Proposition 3.4. Let $\varepsilon>0$ be given. One can find two functions $\alpha$ and $g$ satisfying the assumptions (1.9)-(1.11) and the inequalities

$$
2 \int_{0}^{1} g(s) d s<\left(\max _{y \in \bar{\omega}} \alpha(y)-\min _{y \in \bar{\omega}} \alpha(y)\right)\left(\langle\alpha\rangle-\min _{y=\bar{\omega}} \alpha(y)\right)<2 \int_{0}^{1} g(s) d s+\varepsilon,
$$

such that the corresponding solution $(u, c)$ of $(1.6)-(1.8)$ includes an inversion of the velocity field (i.e. satisfies (1.17)).

Proof. The proof is mainly analogous to the previous one, and uses the asymptotic analysis for high activation energies of the one-dimensional problem (3.12). Referring to [5] for the details, we just recall here that the solution $(\hat{u}, \hat{c})$ of $(3.12)$ always satisfies

$$
2 \int_{0}^{1} g(s) d s<\hat{c}^{2}
$$


This can also be inferred from the proof of Proposition 3.3. Moreover, it is shown in [5] that one can choose $g$ (corresponding to a high activation energy) such that

$$
2 \int_{0}^{1} g(s) d s<\hat{c}^{2}<2 \int_{0}^{1} g(s) d s+\frac{1}{2} \varepsilon .
$$

Having chosen $g$ such that (3.22) holds, we set

$$
\alpha_{1}=\sqrt{2 \int_{0}^{1} g(s) d s+\varepsilon},
$$

and define the sequence of functions $\alpha_{n}$ as in (3.9). Again the corresponding solution $\left(u_{n}, c_{n}\right)$ of (3.1)-(3.4) converges to $\left(\hat{u}, \hat{c}-\alpha_{1}\right)$. For $n$ large enough, $\alpha_{n}$ satisfies (3.20), and $c_{n}$ is strictly negative since $\hat{c}-\alpha_{1}<0$ from (3.22)-(3.23); we can then conclude as in the preceding proof.

Acknowledgement. This work was supported in part by Direction des Recherches, Etudes et Techniques under contract 87-209.

\section{References}

1. S. Agmon, A. Douglis \& L. Nirenberg, "Estimates near the boundary for solutions of elliptic partial differential equations satisfying general boundary conditions I', Comm. Pure Appl. Math. 21, pp. 623-727 (1959).

2. F. Benkhaldoun \& B. LARrouturou, "A finite-element adaptive investigation of curved stable and unstable flame fronts", 76, pp. 119-134 (1989).

3. H. BerestyCKi \& B. LARrouturou, "A semilinear elliptic equation in a strip arising in a two-dimensional flame propagation model", J. für Reine und Angewandte Mathematik, 396, pp. 14-40 (1989).

4. H. BERESTYCKI \& B. LARROUTUROU, "Quelques aspects mathématiques de la propagation des flammes prémélangées", Nonlinear partial differential equations and their applications, Collège de France seminar, Brezis \& Lions, eds., Research Notes in Mathematics, Pitman-Longman, London, to appear.

5. H. BerestyCKI, B. NicolaenKo \& B. ScheURER, "Travelling wave solutions to combustion models and their singular limits", SIAM J. Math. Anal. 16 (6), pp. 1207-1242 (1985).

6. H. BerestyCKI \& L. NirenBerg, "Monotonicity, symmetry and antisymmetry of solutions of semilinear elliptic equations", J. Geom. and Phys. (special issue dedicated to I. M. Gelfand), to appear.

7. H. BERESTYCKI \& L. NIRENBERG, "Some qualitative properties of solutions of semilinear elliptic equations in cylindrical domains, to appear.

8. R, GARDNER, "Existence of multidimensional travelling wave solutions of an initialboundary value problem", J. Diff. Equ. 61, pp. 335-379 (1986).

9. W. E. JoHNson, "On a first-order boundary value problem for laminar flame theory", Arch. Rational Mech. Anal. 13, pp. 46-54 (1963).

10. W. E. Johnson \& W. NACHBAR, "Laminar flame theory and the steady linear burning of a monopropellant", Arch. Rational Mech. Anal. 12, pp. 58-91 (1963).

11. JA.I. KANEL', "Stabilization of solutions of the Cauchy problem for equations encountered in combustion theory", Mat. Sbornik 59, pp. 245-288 (1962). 
12. JA.I. KANEL', "On steady state solutions to systems of equations arising in combustion theory", Dokl. Akad. Nauk USSR 149 (2), pp. 367-369 (1963).

13. B. Larrouturou, "Introduction to combustion modelling", Springer Series in Computational Physics, to appear, (1990).

14. C. M. Lx, Thesis, Courant Institute of Mathematical Sciences, New York-University, in preparation.

15. G. I. SivASHINSKY, "Instabilities, pattern formation and turbulence in flames", Ann. Rev. Fluid Mech. 15, pp. 179-199 (1983).

Laboratoire d'analyse numérique

Université Paris VI

INRIA Sophia-Antipolis

Valbonne, France

Ceremade

Université Paris IX Dauphine

(Received May 2, 1989) 